\title{
Neuromorphic Robot Dream
}

\author{
Alexander Tchitchigin - Max Talanov • Larisa Safina • Manuel Mazzara
}

the date of receipt and acceptance should be inserted later

\begin{abstract}
In this paper we present the next step in our approach to neurobiologically plausible implementation of emotional reactions and behaviors for real-time autonomous robotic systems. The working metaphor we use is the "day and the "night" phases of mammalian life. During the "day phase" a robotic system stores the inbound information and is controlled by a light-weight rule-based system in real time. In contrast to that, during the "night phase" information that has been stored is transferred to a supercomputing system to update the realistic neural network: emotional and behavioral strategies.
\end{abstract}

Keywords robotics, spiking neural networks, artificial emotions, affective computing

\section{Introduction}

From our perspective emotional mechanisms tend to be as valuable for computational systems as for human beings. Without understanding and simulation of emotions the AI-human communication becomes ineffective due to a robotic system making wrong decisions

A. Tchitchigin

Service Science and Engineering Laboratory, Innopolis University and Laboratory of Neurobiology, Kazan Federal University, Russia, E-mail: a.chichigin@innopolis.ru

M. Talanov

Laboratory of Neurobiology, Kazan Federal University, Russia, E-mail: max.talanov@gmail.com

L. Safina

Service Science and Engineering Laboratory, Innopolis University, Russia, E-mail: 1.safina@innopolis.ru

M. Mazzara

Service Science and Engineering Laboratory, Innopolis University, Russia, E-mail: m.mazzara@innopolis.ru and inhumane reactions. However, these phenomena seem to be "difficult" for computational as well as AI and robotics researchers and require to use the simulation of nerobiological processes for implementation, expanding the practical autonomous real-time control of a robotic platform with realistic emotional appraisal and behavior, based on simulation of spiking neural network (sNN) with neuromodulation.

\section{Day phase and Night phase}

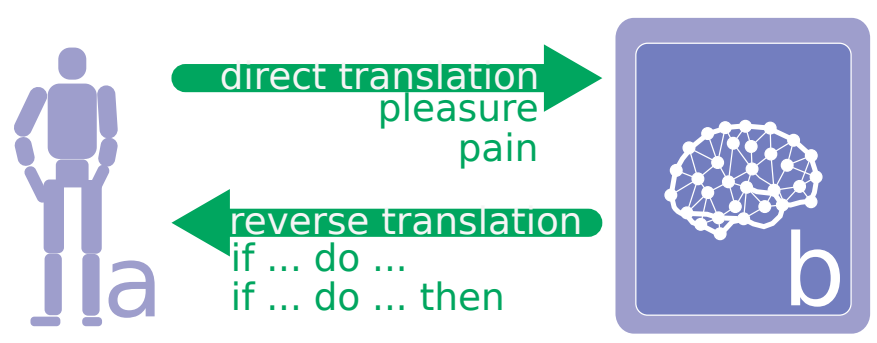

Fig. 1: Neuromorphic robot dream direct and reverse translations

We expect real-time behaviour from a robotic system with traditional rule-based control system. In the "day phase" the control system should react on the input stimuli as well as record and store them for postprocessing during the "night phase". We call the supercomputer sNN simulation that does the processing during the "night phase" the "sleeping brain". When robot transfers its experience via direct translation, the "sleeping brain" processes its inputs, inferring an emotional response of the system from levels of neuromodulators [5]. Emotional responses of the sNN are used 
during the generation of updates to the rules of the control system which are sent back to the robot.

\section{Bisimulation and Neuromorphic simulation}

In our previous paper [4] we have proposed the "bisimulation" mechanism, when we are training the system to adapt to the rules and the structure of the rules of the particular robotic control system.

The other way is to use synchronisation methods introduced on the figure 1 .

When the "sleeping brain" is ready to transfer the updated behavioral strategies back to the robotic system, it starts the procedure that we call "reverse translation". It is based on the mapping of neuronal structures to the rule based description. This mapping was inherited from the Marvin Minsky's book "The emotion machine" 3].

Minsky considers rules of the IF . . DO . THEN . . shape that we associate with cortical columns employing models of the sparse distributed memory [2] and the hierarchical temporal memory [1].

This temporally consecutive activations correspond to the $I F \ldots D O \ldots T H E N \ldots$ rules where the $T H E N$ part represents the expected outcome which is at the same time the condition for the next rule (following the model by Minsky in 3]).

With this approach we could reuse the same "sleeping brain" simulation with the number of simultaneously operating robots. The advantages would be the resource-efficiency (the sNN simulation of a mammalian brain is extremely memory and CPU consuming) and that the "sleeping brain" will receive more information, more examples and will learn faster. Also different robotic systems, possibly performing different tasks in different environments, will benefit from the each other experience via the single-brain configuration.

\subsection{Direct translation}

Considering the initial training of the "sleeping brain" simulation (nurturing period so to say), we propose to decouple from a particular control system . The approach is to perform a translation of raw recorded signals into the appropriate stimulation of brain cells. For instance, a robotic system has an optical camera and records an inbound video stream, the system transforms the stream non-linearly into the stimulation of visual cortex cells during the direct translation, and different sensors and channels will use different mapping functions. The "pain" reaction is activated if the signal on any sensory channel is too strong, triggering the massive neuronal excitation. The proposed mechanism extracts punishment signals flexibly from raw inbound streams of any robotic system.

We have to admit that the mapping of the "pleasure" signals is less clear. One option is to associate the battery level of a robotic system to the sense of the "hunger" this way the "charging" is associated to the feeding thus "pleasure" increasing the dopamine level. Obviously, further research in this direction is needed.

\section{Conclusion}

As the development of our previous ideas [4] we introduce the integration of a robotic system with the simulated "brain" (a spiking neural network). We propose the approach of two translations: robot $\rightarrow s N N$ and $s N N \rightarrow$ robot. The first one ("direct translation") we associate with the animal training and the second one we call "reverse translation", that do not have direct analogy in biological life. Principles of the direct translation are the following: we simulate the sensory input triggering the simulated neurones of proper parts of the brain based on the robotic system "experience" that has been stored, taking into account "pleasure and pain" effects. The reverse translation is based on the multipass transformation and convolution of the temporalprobabilistic rules describing the sNN.

\section{Acknowledgments}

Part of the work was performed according to the Russian Government Program of Competitive Growth of Kazan Federal University.

\section{References}

1. Hawkins, J., Blakeslee, S.: On Intelligence. Henry Holt and Company (2004)

2. Kanerva, P.: Sparse Distributed Memory. A Bradford book. Cambridge, Mass. (1988)

3. Minsky, M.: The Emotion Machine: Commonsense Thinking, Artificial Intelligence, and the Future of the Human Mind. Simon \& Schuster (2007)

4. Tchitchigin, A., Talanov, M., Safina, L., Mazzara, M.: Robot Dream, pp. 291-298. Springer International Publishing, Cham (2016). DOI 10.1007/978-3-319-39883-9 24

5. Vallverdú, J., Talanov, M., Distefano, S., Mazzara, M. Tchitchigin, A., Nurgaliev, I.: A cognitive architecture for the implementation of emotions in computing systems. Biologically Inspired Cognitive Architectures (2015). DOI 10.1016/j.bica.2015.11.002. URL http://www.scopus . com/inward/record.url?eid=2-s2 . 0-84947997777 \{\&\}partnerID=tZOtx3y1http://linkinghub. elsevier.com/retrieve/pii/S2212683X15000663 